\title{
Well-Being and Territory: Methods and Strategies
}

\author{
Matteo Mazziotta ${ }^{1} \cdot$ Chiara Gigliarano $^{2} \cdot$ Stefania Rimoldi ${ }^{3}$
}

Published online: 19 February 2021

(c) The Author(s), under exclusive licence to Springer Nature B.V. part of Springer Nature 2022, corrected publication 2022

\section{Preface}

In the last few years, the measurement of multidimensional phenomena has been one of the most treated research topics; in fact, the interest in the "quantification" of complex events has considerably increased and it has involved several scientific fields. Certainly, Social Indicators Research has been playing a leading role in the international scenario by contributing to the discussion of the entire Scientific Community.

In particular, the measurement of multidimensional well-being has stimulated a vivid debate among scholars from different research fields (economists, philosophers, sociologists and statisticians) and of very distant cultures.

At first researchers debated on well-being as a multidimensional phenomenon; then they moved discussing whether representing well-being with many numbers (a dashboard of indicators) or rather with a single number (a composite index). Finally, they focused their attention on the most robust methodologies for the construction of composite indices. Although the distinction between the three strands of research has characterized the scientific debate, the vast production shows that they have one common element, the territory, as

\footnotetext{
Unfortunately, the following articles were by mistake included in other issues but are part of the Special Issue on Well-Being and Territory: Methods and Strategies "Tomaselli, V., Giuffrida, G., Gozzo, S. et al. Building Decision-making indicators Through Network Analysis of Big Data. Soc Indic Res 151, 33-49 (2020). https://doi.org/10.1007/s11205-020-02363-2". "Crocetta, C., Antonucci, L., Cataldo, R. eta al. Higher-Order PLS-PM Approach for Different Types of Constructs. Soc Indic Res 154, 725-754 (2021). https://doi.org/10.1007/s11205-020-02563-w". "Greco, F., Polli, A. Security Perception and People Well-being. Soc Indic Res 153, 741-758 (2021). https://doi.org/10.1007/s11205-020-02341-8". "Riniolo, V., Ortensi, L.E. Young Generations' Activism in Italy: Comparing Political Engagement and Participation of Native Youths and Youths from a Migrant Background. Soc Indic Res 153, 923-955 (2021). https://doi.org/10.1007/s11205-020-02487-5".
}

Matteo Mazziotta mazziott@istat.it

Chiara Gigliarano chiara.gigliarano@uninsubria.it

Stefania Rimoldi stefania.rimoldi@unimib.it

1 Italian Institute of Statistics, Rome, Italy

2 University of Insubria, Varese, Italy

3 University of Milano - Bicocca, Milan, Italy 
the way to contextualize the measurement of well-being in places where people live, where citizens are the actors of civil society and where progress is pushing the development of human capability. Indeed, well-being strongly depends on people's actions and behaviours in the territory. Thanks to the increasing availability of administrative sources and Big Data it is nowadays possible to compute indicators at a very disaggregated territorial level and, therefore, to measure well-being for very small geographical areas.

For more than eighty years, the Italian Society of Economics, Demography and Statistics (SIEDS) ${ }^{1}$ has been focused on these issues by promoting multidisciplinary scientific debate and welcoming the research ideas of scholars with different skills and professional experiences.

This Special Issue collects a selection of papers presented by SIEDS members during the LVI Scientific Meeting in Ascoli Piceno (Italy) on May 23-24, 2019. The thirty papers published offer an interesting overview on the measurement of well-being from a multidisciplinary point of view, which represents one of the prerogatives of both Social Indicators Research and the Italian Society of Economics, Demography and Statistics.

This Special Issue offers an interesting contribution to the literature on this topic, as the editors intended to offer a collection of the avant-garde research in the different scientific approaches that characterize the SIEDS. Many papers deal with strictly methodological aspects mainly related to the theory of composite indicators, while others present models for territorial classification. Several papers focus on the socio-economic and environmental components of the multidimensional phenomenon, while others direct their attention on new data sources for measuring well-being. Some papers are based on objective indicators linked to the main macroeconomic variables, while others stress the importance of subjective indicators for the perception of the phenomenon. Finally, some works focus on the role of the various factors that affect the well-being of individuals at different stages of life. The plurality of ideas and approaches represents the most important feature of scientific research, especially when the issue is as complex as that of well-being.

We hope that this book may represent a valuable contribution to the socio-economic literature and may inspire many more scholars towards continuous development of theories and methodologies for measuring well-being at territorial level.

Publisher's Note Springer Nature remains neutral with regard to jurisdictional claims in published maps and institutional affiliations.

1 wwW.sieds.it. 